\title{
GEÖCZE AREA AND A CONVERGENCE PROPERTY
}

\author{
RONALD GARIEPY
}

Abstract. Suppose $f$ is a continuous mapping with finite Lebesgue area from a polyhedral region $X \subset R^{k}$ into $R^{n}, 2 \leqq k \leqq n$. Let $f=l \circ m$ be the monotone-light factorization of $f$ with middle space $M$.

If $f$ satisfies a "cylindrical condition" considered by T. Nishiura, then a current valued measure $T$ over $M$ can be associated with $f$ by means of the Cesari-Weierstrass integral, and if $\left\{f_{i}\right\}$ is any sequence of quasi-linear maps $f_{i}: X \rightarrow R^{n}$ converging uniformly to $f$ with bounded areas, then

$$
T(g)(\phi)=\lim _{i \rightarrow \infty} \int_{X}(g \circ m) f_{i}^{\#} \phi
$$

whenever $\phi$ is an infinitely differentiable $k$-form in $R^{n}$ and $g$ is a continuous real valued function on $M$ which vanishes on $m$ (Bdry $X$ ).

The total variation measure of $T$, taken with respect to mass, coincides with the Geöcze area measure over $M$.

1. Suppose $f$ is a continuous mapping with finite Lebesgue area from a polyhedral region $X \subset R^{k}$ into $R^{n}, 2 \leqq k \leqq n$. Let $f=l \circ m$ denote the monotone-light factorization of $f$ with middle space $M$.

Suppose $p: R^{n} \rightarrow R^{k}$ is an orthogonal projection. Let $p \circ f=\bar{l} \circ \bar{m}$ denote the monotone-light factorization of $p \circ f$ with middle space $\bar{M}$ and let $h$ be the monotone map such that the diagram

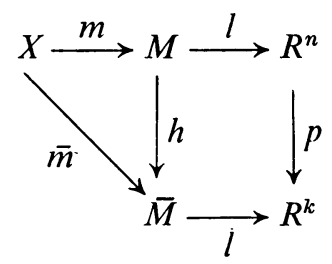

commutes.

Let $C_{p}=M \cap\left\{z: \operatorname{diam} h^{-1}(h(z))>0\right\}$, where diameter is taken with respect to the usual metric in $M$. It was shown in [N1, 2.1] that, if either $k=2$ or the $k+1$ dimensional Hausdorff measure of $f(X)$ is zero,

Received by the editors April 16, 1971.

AMS 1970 subject classifications. Primary 26A63, 28A75.

(c) American Mathematical Society 1972 
then the $k$ dimensional Lebesgue measure of $p \circ l\left(C_{p}\right)$ is zero for every orthogonal projection $p$.

Let $\Lambda(k, n)$ denote the set of all $k$-tuples $\lambda=\left(\lambda_{1}, \cdots, \lambda_{k}\right)$ of integers such that $1 \leqq \lambda_{1}<\cdots<\lambda_{k} \leqq n$ and let $p^{\lambda}: R^{n} \rightarrow R^{k}$ be the orthogonal projection defined by

$$
p^{\lambda}(y)=\left(y_{\lambda_{1}}, \cdots, y_{\lambda_{k}}\right) \text { for } y=\left(y_{1}, \cdots, y_{n}\right) \in R^{n} .
$$

Let $C(M)$ denote the space of all continuous real valued functions on $M$ and let $C_{0}(M)$ denote the space of those $g \in C(M)$ which vanish on $m($ Bdry $X)$.

Let $e_{1}, \cdots, e_{n}$ be the standard basis in $R^{n}$ and let $e_{\lambda}=e_{\lambda_{1}} \wedge \cdots \wedge e_{\lambda_{k}}$, $\lambda \in \Lambda(k, n)$, be the corresponding basis for the space of $k$-vectors in $R^{n}$.

For $\lambda \in \Lambda(k, n)$ let $f^{\lambda}=p^{\lambda} \circ f$ and for each simple polyhedral region $\pi \subset X$ let

$$
u\left(f^{\lambda}, \pi\right)=\int_{R^{k}} O\left(f^{\lambda}, \pi, y\right) d y
$$

where $O\left(f^{\lambda}, \pi, y\right)$ denotes the topological index of $y \in R^{k}$ with respect to the mapping $f^{\lambda} \mid \pi$ if $y \in R^{k} \mid f^{\lambda}(\operatorname{Bdry} \pi)$ and $O\left(f^{\lambda}, \pi, y\right)=0$ if $y \in f^{\lambda}(\operatorname{Bdry} \pi)$. Let

$$
u(f, \pi)=\sum_{\lambda \in \Lambda(k, n)} u\left(f^{\lambda}, \pi\right) e_{\lambda} .
$$

For any finite nonoverlapping collection $P$ of simple polyhedral regions $\pi \subset X$ let

$$
\begin{aligned}
\delta(P)= & \max \{\operatorname{diam} f(\pi): \pi \in P\} \\
& +\max \left\{V\left(f^{\lambda}\right)-\sum_{\pi \in P}\left|u\left(f^{\lambda}, \pi\right)\right|: \lambda \in \Lambda(k, n)\right\}
\end{aligned}
$$

where $V\left(f^{\lambda}\right)$ is the Geöcze area of $f^{\lambda}$.

In [G, Theorem 1] it was shown that if the infimum of the numbers $\delta(P)$, taken over all $P$ as above, is zero, then the Cesari-Weierstrass integral

$$
T(g)(\phi)=\lim _{\delta(P) \rightarrow 0} \sum_{\pi \in P} \frac{1}{|\pi|} \int_{\pi} g \circ m(x) \phi(f(x)) \cdot u(f, \pi) d x
$$

exists whenever $g \in C(M)$ and $\phi$ is an infinitely differentiable $k$-form on $R^{n}$. Here $|\pi|$ denotes the $k$ dimensional Lebesgue measure of $\pi$. The current valued linear mapping $T$ on $C(M)$ possesses a unique extension to the class of all bounded Borel measurable functions on $M$ such that Lebesgue's bounded convergence theorem holds. 
THEOREM. If the $k$ dimensional Lebesgue measure of $p^{\lambda} \circ l\left(C_{p^{\lambda}}\right)$ is zero for each $\lambda \in \Lambda(k, n)$, then:

(1) A current valued measure $T$ can be associated with $f$ by means of the Cesari-Weierstrass integral.

(2) If $\left\{f_{i}\right\}$ is any sequence of quasi-linear mappings $f_{i}: X \rightarrow R^{n}$ converging uniformly to $f$ with bounded areas, then

$$
T(g)(\phi)=\lim _{i \rightarrow \infty} \int_{X}(g \circ m) f_{i}^{\#} \phi
$$

whenever $g \in C_{0}(M)$ and $\phi \in E^{k}\left(R^{n}\right)$.

(3) The total variation measure $\|T\|$ of $T$, taken with respect to mass, coincides with the Geöcze area measure over $M$.

The notation is that of $[\mathbf{F 1}]$ and $[\mathbf{G}]$.

In case either $k=2$ or the $k+1$ dimensional Hausdorff measure of $f(X)$ is zero, the conclusions of the Theorem above can be obtained using the results of $[\mathbf{F 1}]$ and $[\mathbf{G}]$. It is the purpose of this note to show that the given weaker hypothesis suffices; in particular that one can avoid the use of [G, Theorem 5].

Conclusion (2) of the Theorem should be compared with [F1, 3.10]. Note that $f|X| m^{-1}(m($ Bdry $X))$ need not be proper

2. The case $k=n$. Suppose $f$ is a continuous mapping with finite Lebesgue area from a polyhedral region $X \subset R^{k}$ into $R^{k}, 2 \leqq k$. By [G, Theorems 1 and 2] applied with $k=n$, the current valued measure $T$ associated with $f$ by means of the Cesari-Weierstrass integral exists and the total variation measure $\|T\|$ of $T$ coincides with the Geöcze area measure over $M$.

The arguments of [F1, 3.4 and 3.6] show that there exists a unique current valued measure $S$ over $M \backslash m($ Bdry $X)$ such that, for every sequence $\left\{f_{i}\right\}$ of quasi-linear maps $f_{i}: X \rightarrow R^{k}$ converging uniformly to $f$ with bounded areas, we have

$$
S(g)(\phi)=\lim _{i \rightarrow \infty} \int_{X}(g \circ m) f_{i}^{\#} \phi
$$

for $g \in C_{0}(M)$ and $\phi \in E^{k}\left(R^{k}\right)$.

Since the Lebesgue area of $f$ and the Geöcze area of $f$ are equal when $k=n$, there exists a sequence $\left\{f_{i}\right\}$ of quasi-linear maps converging uniformly to $f$ with areas converging to the Geöcze area of $f$. Application of [G, Theorem 4] to this case shows that

$$
T(g)(\phi)=\lim _{i \rightarrow \infty} \int_{X}(g \circ m) f_{i}^{\#} \phi
$$

for $g \in C_{0}(M)$ and $\phi \in E^{k}\left(R^{k}\right)$. 
3. Proof of the Theorem. Suppose $\left\{f_{i}\right\}$ is any sequence of quasi-linear maps converging uniformly to $f$ with bounded areas. As in [F1, 3.4] there is a subsequence, which we continue to denote by $\left\{f_{i}\right\}$, such that

$$
S(g)(\phi)=\lim _{i \rightarrow \infty} \int_{X}(g \circ m) f_{i}^{\#} \phi
$$

exists whenever $g \in C(M)$ and $\phi \in E^{k}\left(R^{n}\right)$. The current valued mapping $S$ so defined possesses a unique extension, which we continue to denote by $S$, to the class of all bounded Borel measurable functions on $M$ such that Lebesgue's bounded convergence theorem holds. With obvious modifications, the argument of $[\mathbf{F 1}, 3.4]$ shows that $S(B)$ is a rectifiable current whenever $B$ is a Borel subset of $M \backslash m($ Bdry $X)$.

From [N1] we see that the infimum of the numbers $\delta(P)$, taken over all finite collections $P$ of nonoverlapping simple polyhedral regions $\pi \subset X$, is zero and hence we can define [G, Theorem 1] a current valued function $T$ on $C(M)$ by letting

$$
T(g)(\phi)=\lim _{\delta(P) \rightarrow 0} \sum_{\pi \in P} \frac{1}{|\pi|} \int_{\pi} g \circ m(x) \phi(f(x)) \cdot u(f, \pi) d x
$$

whenever $g \in C(M)$ and $\phi \in E^{k}\left(R^{n}\right)$. We denote by $T$ also the unique extension of this current valued mapping to the class of all bounded Borel measurable functions on $M$ such that Lebesgue's bounded convergence theorem holds.

Let $e^{1}, \cdots, e^{n}$ denote the usual basis for the space of covectors in $R^{n}$ and let $e^{\lambda}=e^{\lambda_{1}} \wedge \cdots \wedge e^{\lambda_{k}}, \lambda \in \Lambda(k, n)$, denote the corresponding basis for the space of $k$-covectors in $R^{n}$.

Let $u \in E^{0}\left(R^{n}\right)$ be such that $u(x)=1$ for $x \in R^{n}$ and, for $\lambda \in \Lambda(k, n)$, consider the signed Borel measures defined over $M$ by letting

$$
S^{\lambda}(B)=S(B) \wedge e^{\lambda}(u) \text { and } T^{\lambda}(B)=T(B) \wedge e^{\lambda}(u)
$$

whenever $B$ is a Borel subset of $M$.

In order to prove statement (2) it suffices to show that $S^{\lambda}(B)=T^{\lambda}(B)$ whenever $B$ is a Borel subset of $M \backslash m($ Bdry $X)$.

For fixed $\lambda \in \Lambda(k, n)$ let $l_{\lambda} \circ m_{\lambda}$ denote the monotone-light factorization of $f^{\lambda}=p^{\lambda} \circ f$ with middle space $M_{\lambda}$ and let $h$ be the monotone map such that the following diagram commutes:

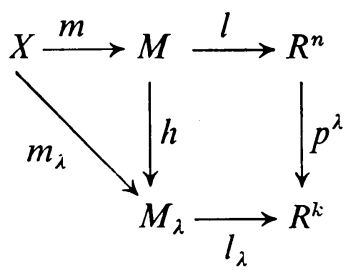


From the case $k=n$, previously considered, we have $S^{\lambda}(\psi \circ h)=T^{\lambda}(\psi \circ h)$ whenever $\psi \in C_{0}\left(M_{\lambda}\right)$. By Lebesgue's bounded convergence theorem the above equality holds for any bounded Borel measurable function $\psi$ on $M_{\lambda}$ that vanishes on $m_{\lambda}($ Bdry $X)$. In particular

$$
S^{\lambda}\left(h^{-1}(B)\right)=T^{\lambda}\left(h^{-1}(B)\right)
$$

whenever $B$ is a Borel subset of $M_{\lambda} \mid m_{\lambda}(\operatorname{Bdry} X)$.

For any Borel set $A \subset M \backslash\left[C_{p^{\lambda}} \cup m(\right.$ Bdry $\left.X)\right]$, we have

$$
h(A) \subset M_{\lambda} \mid m_{\lambda}(\operatorname{Bdry} X) \text { and } h^{-1}(h(A))=A .
$$

Hence $S^{\lambda}(A)=T^{\lambda}(A)$.

We will complete the proof of statement (2) by showing that $T^{\lambda}(B)$ and $S^{\lambda}(B)$ both vanish for any Borel set $B \subset C_{p^{\lambda}} \mid m($ Bdry $X)$.

Let $\mu_{\lambda}$ denote the finite Borel measure [N2,6.10] defined over $M_{\lambda}$ by letting

$$
\mu_{\lambda}(B)=\inf \left\{V\left(f^{\lambda} \mid m_{\lambda}^{-1}(U)\right): U \text { open in } M_{\lambda}, U \supset B\right\}
$$

whenever $B$ is a Borel subset of $M_{\lambda}$. Here $V\left(f^{\lambda} \mid m_{\lambda}^{-1}(U)\right)$ denotes the Geöcze area of $f^{\lambda} \mid m_{\lambda}^{-1}(U)$.

For $\eta>0$ let $C^{\eta}=M \cap\left\{z: \operatorname{diam} h^{-1}(h(z)) \geqq \eta\right\}$. Then each $C^{\eta}$ is closed and $C_{p} \lambda=\bigcup_{\eta>0} C^{\eta}$.

Suppose $\eta>0$ and $P$ is a finite collection of nonoverlapping simple polyhedral regions $\pi \subset X$ with $\delta(P)<\eta$. Then, for each $\pi \in P$, we have

$$
m_{\lambda}(\pi) \cap h\left(C^{\eta}\right) \subset m_{\lambda}(\operatorname{Bdry} \pi) .
$$

By $[\mathbf{N 2}, 6.5], V\left(f^{\lambda} \mid \pi\right)=\mu_{\lambda}\left(m_{\lambda}(\pi)-m_{\lambda}(\operatorname{Bdry} \pi)\right)$ and hence

$$
\begin{aligned}
\mu\left(M_{\lambda}\right)=V\left(f^{\lambda}\right) & \leqq \sum_{\pi \in P} V\left(f^{\lambda} \mid \pi\right)+\delta(P) \\
& =\sum_{\pi \in P} \mu_{\lambda}\left(m_{\lambda}(\pi)-m_{\lambda}(\text { Bdry } \pi)\right)+\delta(P) \\
& \leqq \mu_{\lambda}\left(M_{\lambda} \mid h\left(C^{\eta}\right)\right)+\delta(P) .
\end{aligned}
$$

Thus $\mu_{\lambda}\left(h\left(C^{\eta}\right)\right)=0$ for each $\eta>0$ and hence $\mu_{\lambda}\left(h\left(C_{p} \lambda\right)\right)=0$.

If $U$ is open in $M_{\lambda}$, then $h^{-1}(U)$ is open in $M$ and $m_{\lambda}^{-1}(U)=m^{-1}\left(h^{-1}(U)\right)$. Thus

$$
\inf \left\{V\left(f^{\lambda} \mid m^{-1}(U)\right): U \text { open in } M, U \supset C_{p^{\lambda}}\right\}=0 .
$$

(This argument was suggested by T. Nishiura [N3, 4.5].) Since

$$
T^{\lambda}(\psi)=\lim _{\delta(P) \rightarrow 0} \sum_{\pi \in P} \frac{1}{|\pi|} \int_{\pi} \psi \circ m(x) u\left(f^{\lambda}, \pi\right) d x
$$

for $\psi \in C(M)$, we infer that $T^{\lambda}(B)=0$ whenever $B$ is a Borel subset of $C_{p^{\lambda}}$. 
If $B$ is a Borel subset of $C_{p} \lambda \mid m(\operatorname{Bdry} X)$ whose closure is contained in $C_{p^{\lambda}}$, then $S(B)$ is rectifiable and spt $S(B) \subset l\left(C_{p^{\lambda}}\right)$. Thus, by [F2, 2.1], $S^{\lambda}(B)=0$. Since $C_{p^{\lambda}}$ is an $F_{\sigma}$ set, we have $S^{\lambda}(B)=0$ for each Borel set $B \subset C_{p^{\lambda}} \mid m($ Bdry $X$ ) and statement (2) follows.

Statement (3) now follows from [G, Theorems 3 and 7].

4. Example. We consider here an example from [N1, 2.2] to show that, in case $2<k<n$, the hypothesis of the Theorem may be satisfied even though the $k+1$ dimensional measure of $f(X)$ is positive.

Let $X_{0}$ denote the unit square in $R^{2}$ and let $\bar{f}$ denote a continuous mapping with finite area from $X_{0}$ into $R^{3}$ such that $\bar{f}\left(X_{0}\right)$ has positive 3 dimensional measure. If $\bar{p}: R^{3} \rightarrow R^{2}$ is any orthogonal projection and $\bar{l}$ is the light factor of $\bar{f}$, then the 2 dimensional measure of $\bar{p} \circ \bar{l}\left(C_{\bar{p}}\right)$ is zero.

Let $X=X_{0} \times I$ where $I$ is the unit interval and let $f$ denote the continuous mapping with finite Lebesgue area from $X$ into $R^{4}$ defined by letting

$$
f(x)=\left(\bar{f}\left(x_{1}, x_{2}\right), x_{3}\right) \text { for } x=\left(x_{1}, x_{2}, x_{3}\right) \in X .
$$

The 4 dimensional measure of $f(X)$ is positive. If $p: R^{4} \rightarrow R^{3}$ is an orthogonal projection such that $p\left(e_{4}\right)=0$ and $l$ is the light factor of $f$, then the 3 dimensional measure of $p \circ l\left(C_{p}\right)$ is equal to that of $\bar{f}\left(X_{0}\right)$ and hence is positive. If, however, $p\left(e_{4}\right) \neq 0$, then it is readily seen that the 3 dimensional measure of $p \circ l\left(C_{p}\right)$ is zero.

\section{REFERENCES}

[C1] L. Cesari, Quasi additive set functions and the concept of integral over a variety, Trans. Amer. Math. Soc. 102 (1962), 94-113. MR 26 \#292.

[C2] — Extension problem for quasi-additive set functions and Radon-Nikodym derivatives, Trans. Amer. Math. Soc. 102 (1962), 114-146. MR 26 \#293.

[F1] H. Federer, Currents and area, Trans. Amer. Math. Soc. 98 (1961), 204-233. MR 23 \#A1006.

[F2] - Some theorems on integral currents, Trans. Amer. Math. Soc. 117 (1965), 43-67. MR 29 \#5984.

[G] R. Gariepy, Current valued measures and Geöcze area, Trans. Amer. Math. Soc. 166 (1972), 133-146.

[N1] T. Nishiura, The Geöcze $k$-area and a cylindrical property, Proc. Amer. Math. Soc. 12 (1961), 795-800. MR 23 \#A3238.

[N2] - Integrals over a product variety and Fubini theorems, Rend. Circ. Mat. Palermo (2) 24 (1965), 207-236. MR 33 \#5848.

[N3] — Area measures and Radó's lower area, Trans. Amer. Math. Soc. 159 (1971), 355-367.

Department of Mathematics, University of Kentucky, Lexington, Kentucky 40506 